Reprod. Nutr. Dévelop. 1980, 20 (2), 545-552.

\title{
Mécanismes du contrôle de la synthèse de l'ovalbumine et de la conalbumine dans l'oviducte de poulet par l'œstradiol et la progestérone. Etudes du récepteur de l'œstradiol et des effets d'un anti-œstrogène, le tamoxifen.
}

par Nadine BINART, Maria-Grazia CATELLI, Claudine GEYNET, R. L. SUTHERLAND *, J. MESTER, E.-E. BAULIEU

INSERM U. 33, Laboratoire Hormones 94270 Bicêtre, France.

* Ludwig Institute for Cancer Research, University of Sydney, NSW 2006, Australie.

Summary. Control of ovalbumin and conalbumin synthesis in the chick oviduct by estradiol and progesterone : Estradiol receptor and effects of an antiestrogen, tamoxifen.

1. Nuclear estrogen receptor levels in the chick oviduct at $1 \mathrm{~h}$ after injection of estradiol were dose-dependent up to $0.1 \mathrm{mg} / \mathrm{kg}$ and dose-independent within a range of 0.1 to $10 \mathrm{mg} / \mathrm{kg}$.

2. Early induction kinetics of ovalbumin (up to $6 \mathrm{~h}$ ) and conalbumin (up to $2 \mathrm{~h}$ ) by estradiol benzoate $\left(E_{2} b\right)$ were different for each protein, but independent of the dose injected if $>$ $0.1 \mathrm{mg} / \mathrm{kg}$. This result indicated a correlation between the amplitude of estrogenic effect and nuclear receptor concentration.

3. Induction kinetics of both ovalbumin and conalbumin synthesis by progesterone were different than in the case of estrogen, but the early kinetics were again correlated with the nuclear progesterone receptor level $1 \mathrm{~h}$ after the treatment.

4. With the exception of ovalbumin synthesis, all estrogen-induced responses were inhibited when progesterone was injected simultaneously with estradiol during the early time periods (up to $\simeq 6 \mathrm{~h}$ ). Subsequently, the amplitude of induction increased up to and above that induced by estrogen alone.

5. Tamoxifen (trans-1-(p-dimethylaminoethylphenyl)-1,2-diphenylbut-1-ene) inhibited the estrogenic induction of both proteins. The half-effective dose was the same for ovalbumin and conalbumin ( $1 \mathrm{mg} / \mathrm{kg}$ of tamoxifen given simultaneously with $1 \mathrm{mg} / \mathrm{kg}$ of $\mathrm{E}_{2} \mathrm{~b}$ ). Retarded administration of a large dose $(10 \mathrm{mg} / \mathrm{kg})$ of tamoxifen caused a virtually immediate arrest of the induction of conalbumin synthesis. Conversely, the tamoxifen effect could be reversed by a secondary injection of $E_{2} b$.

6. Simultaneous treatment with progesterone plus tamoxifen surprisingly led to a greater induction of both ovalbumin and conalbumin than progesterone alone.

Au cours de la dernière décennie, plusieurs groupes ont étudié la synthèse des protéines du blanc de l'œuf dans l'oviducte de poulet $(1,2,3,4,5)$. L'œstradiol et la progestérone augmentent la synthèse de l'ovalbumine et de la conalbumine, les deux protéines les plus importantes quantativement, et comme la synthèse de ces deux pro- 


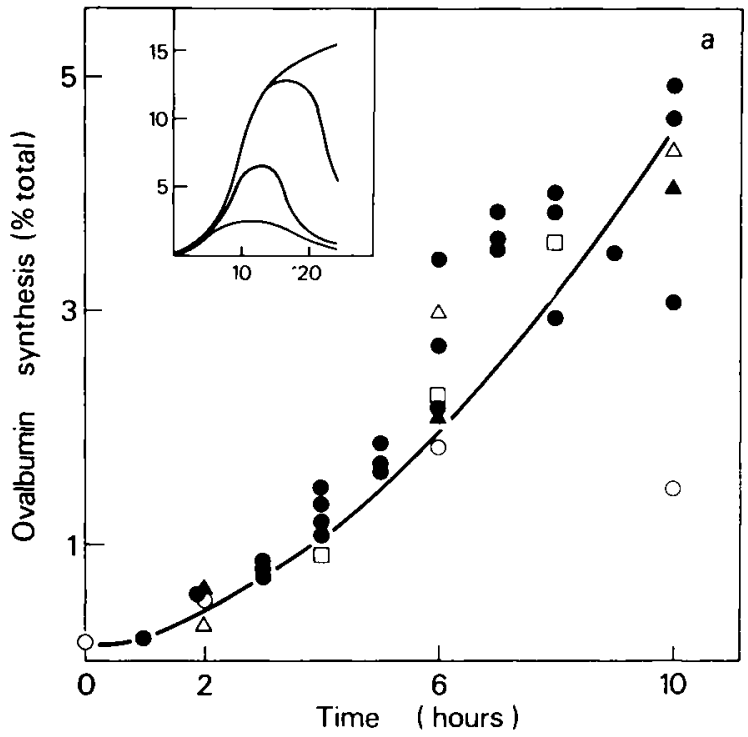

FIG. $1 \mathrm{~A}$.

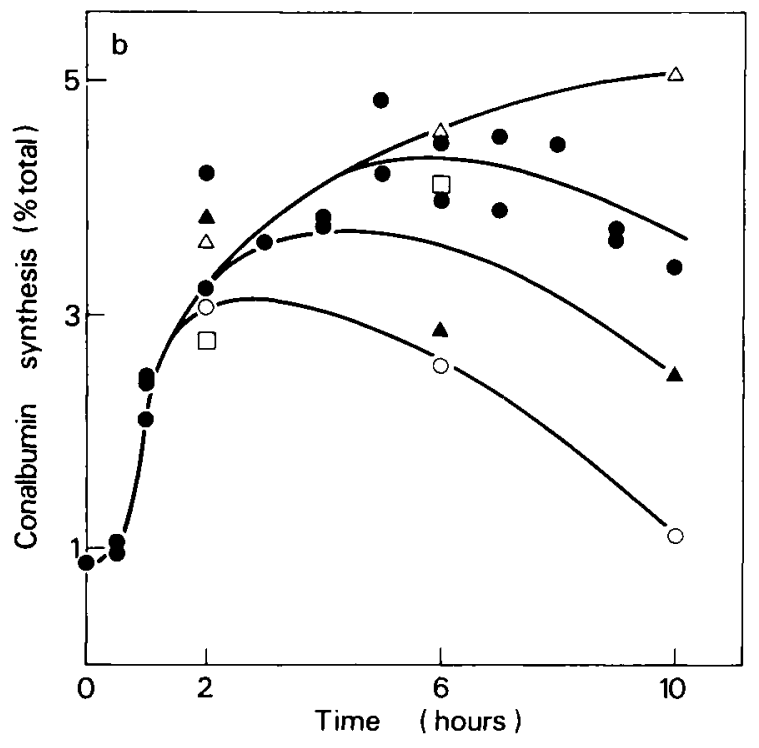

FIG. 1B.

FIG. 1. - Effet de doses différentes d'œestradiol benzoate et de 11ß-méthoxy-17 $\alpha$-éthynyl œstradiol (R 2858) sur le taux de synthèse de l'ovalbumine et de la conalbumine. Des groupes de poulets « withdrawn » depuis 5 semaines reçoivent une injection intramusculaire d'œstradiol, et 3 animaux par point sont sacrifiés aux temps indiqués après l'injection.

A) Taux relatif de synthèse de l'ovalbumine après l'œstradiol benzoate $0-0,0,1 \mathrm{mg}, \Delta-\Delta, 0,5 \mathrm{mg}$, $\bullet-1 \mathrm{mg}, \Delta-\Delta 3 \mathrm{mg}$, et le R $28581 \mathrm{mg} / \mathrm{kg}$ 口- . L'encadré représente la synthèse de l'ovalbumine pendant une période de $24 \mathrm{~h}$.

B) Taux relatif de synthèse de la conalbumine après l'œstradiol benzoate et $R$ 2858. Les symboles sont les mêmes qu'en $A$. 
téines se fait, d'après les résultats d'études histo-immunologiques (6), exclusivement dans les cellules tubulaires, le système constitue à cet égard un exemple intéressant du contrôle de la synthèse de protéines spécifiques par deux hormones dans la même cellule. Les propriétés des récepteurs étudiés dans notre laboratoire $(7,8)$ et ailleurs $(9$, 10 ) et les arguments cinétiques de l'induction $(4,11,12)$ indiquent que les deux hormones agissent chacune via leur propre récepteur. Il est particulièrement intéressant par conséquent d'examiner en détail les particularités de la synthèse de chacune des deux protéines, de définir les corrélations possibles avec la distribution subcellulaire des récepteurs, et d'examiner les effets synergiques et antagonistes possibles des deux stéroïdes ainsi que les conséquences de l'administration d'un anti-œstrogène, le tamoxifen (13). Les résultats qui sont présentés ci-dessous sont extraits de deux manuscrits en cours de publication $(14,15)$.

Les études ont été faites sur des poulets (Warren), ayant reçu à l'âge d'une semaine et quotidiennement pendant 10 jours $1 \mathrm{mg}$ d'cestradiol benzoate.

Après suspension du traitement œstrogénique pendant 4 à 6 semaines, les animaux « withdrawn » reçoivent les hormones étudiées. Dans ces conditions, l'oviducte s'est différencié (poids environ $100 \mathrm{mg}$ ) et environ 15 p. 100 de ses cellules sont tubulaires. On mesure le récepleur de l'œstradiol dans les fractions cytosoluble et nucléaire selon les méthodes décrites préalablement (7). Le nombre de sites par cellule est calculé sur la base de la mesure de l'ADN (mais ce calcul ne tient pas compte des 15 p. 100 des cellules des glandes tubulaires, puisque toutes les cellules de l'organe contiennent du récepteur de l'œestradiol). La mesure des taux relatifs de synthèse de l'ovalbumine ef la conalbumine se fait selon les principes établis par Palmiter (16) et détaillés ailleurs (15).

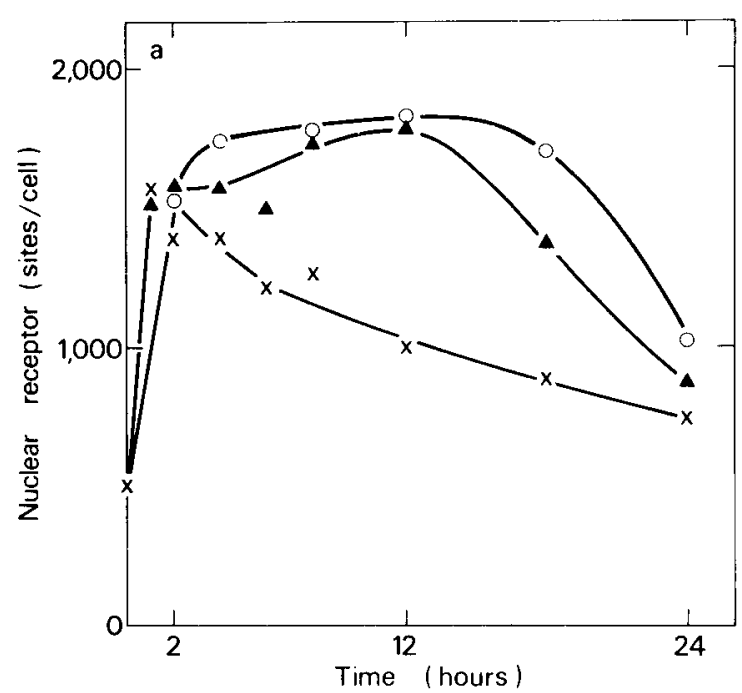

FIG. 2. - Effet de l'œstradiol benzoate et de ( $R$ 2858) sur la concentration du récepteur nucléaire des œstrcgènes. Des poulets « withdrawn 》 reçoivent une injection intramusculaire d'œstradiol benzoate ou de R 2858 dans le propylène glycol. Des groupes de 3 animaux sont sacrifiés aux temps indiqués ef la concentration du récepteur nucléaire de l'œstrogène mesurée après $100 \mathrm{mg} / \mathrm{kg}$

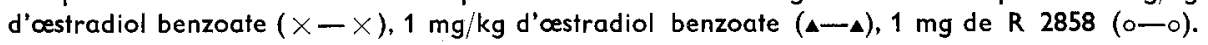


Les principaux résultats nouveaux indiquent:

1) Comme on peut le voir dans la figure 1 ( $A$ et $B$ ), les faux de synthèse de l'ovalbumine ef de la conalbumine par l'œstradiol dépendent de la dose d'hormone injectée, sauf pour les temps précoces. Au contraire, l'induction est indépendante de la dose jusqu'à $6 \mathrm{~h}$ pour l'ovalbumine et $2 \mathrm{~h}$ pour la conalbumine, les courbes divergent ensuite.

2) Si l'on considère les résultats obtenus en mesurant le récepteur de l'œestradiol après administration des différentes doses d'œstradiol benzoate, et de $11 \beta$-méthoxy-17 $\alpha$ éthynyl-œstradiol ( $R$ 2858) qui est un œstrogène de synthèse particulièrement actif (fig. 2), on peut établir que l'amplitude et la durée de la réponse, en taux relatif de synthèse protéiques reflet de l'accumulation des ARN-messagers $(11,17)$, sont d'autant plus importantes que le récepteur nucléaire reste plus longtemps élevé. On sait que, avant l'injection hormonale, le récepteur est distribué pour $2 / 3$ dans le cytoplasme et $1 / 3$ dans le noyau. Sous l'effet de doses supérieures à $0,1 \mathrm{mg} / \mathrm{kg}$ d'œstradiol benzoate, au bout de 1 h 80 p. 100 du récepteur sont mesurés dans la fraction nucléaire. La persistance du récepteur dans le noyau est d'auiant plus longue que la dose est élevée. Cette corrélation entre l'amplitude de la réponse ef la présence du récepteur dans le noyau confirme, dans ce cas précis, ce qui a été antérieurement établi au niveau de l'utérus de rat (test utérotrophique).

3) Des études préalables ont indiqué que les doses d'œstradiol benzoate utilisées ici, supérieures à $0,1 \mathrm{mg} / \mathrm{kg}$, conduisent en $1 \mathrm{~h}$ à un taux identique de récepteur nucléaire. Les doses inférieures à $0,1 \mathrm{mg} / \mathrm{kg}$, au contraire, ne permettent pas cette augmentation maximale (12). Il y a donc corrélation aux temps précoces entre la cinétique d'induction de l'ovalbumine et de la conalbumine et le taux constant de récepteur nucléaire, à toutes les doses d'œstrogène.

4) Les expériences faites en administrant différentes doses de progestérone ont permis des conclusions tout à fait parallèles à celles obtenues après administration d'œstradiol benzoate. En effet, selon les doses, l'importance de la réponse sur chacune des protéines esi d'autant plus grande que la dose administrée $\epsilon s t$ élevée. Le début de l'induction esi semblable dans tous les cas où la progestérone est $>0,5 \mathrm{mg} / \mathrm{kg}$, alors qu'au contraire l'induction est moindre après $0,1 \mathrm{mg}$; cela coïncide avec le niveau maximum de récepteur nucléaire de la progestérone, observé après $1 \mathrm{~h}$ de traitement ef pour des doses $\geqslant 0,5 \mathrm{mg} / \mathrm{kg}(7)$.

5) Quand l'œstradiol benzoate $(1 \mathrm{mg} / \mathrm{kg})$ et la progestérone $(3 \mathrm{mg} / \mathrm{kg})$ sont injectés simultanément, la synthèse de conalbumine est plus faible pendant environ $8 \mathrm{~h}$ que celle observée chez les poulets injectés seulement avec l'œstradiol benzoate. Plus tard, au contraire, le taux de synthèse de la conalbumine rejoint ef dépasse celui observé après l'administration d'œstrogène seul. Ce double phénomène, freinage suivi de dépassement, se retrouve égalemeni quand on administre la progestérone à des temps différents $(6,12,18 \mathrm{~h})$ après l'œstrogène. D'autres paramètres de la réponse aux œstrogènes subissent ces mêmes modifications, par exemple, la concentration du récepleur de l'œstradiol lui-même (cytoplasmique et nucléaire). Les mécanismes moléculaires en jeu dans cet effet de la progestérone sur toute une série d'éléments induits par l'œstrogène, n'ont pas reçu encore d'explications. Il est intéressant de noter que le taux de synthèse de l'ovalbumine après l'injection simultanée d'œstradiol et de proges- 
térone est à tout moment égal à la somme de l'effet de chaque hormone. Cette exception n'a pas non plus reçu d'explications précises.

6) Le tamoxifen est un anti-œstrogène efficace : il inhibe l'effet de l'œstradiol sur la synthèse de l'ovalbumine et de la conalbumine. Son effet dépend de la dose :1 $\mathrm{mg} / \mathrm{kg}$ de tamoxifen conduit à une inhibition de 50 p. 100 de l'effel œstrogénique sur chacune des deux protéines. L'intérêt particulier du tamoxifen dans le système est son absence d'effet œstrogénique au niveau de l'organe cible, même après administration de ce composé, pendant plusieurs jours à la dose de $10 \mathrm{mg}$ par $\mathrm{kg}$ et par jour. L'utilisation de tous les autres anti-œstrogènes dans tous les systèmes biologiques indique qu'ils sont partiellement agonistes (œstrogènes) ; d'ailleurs, même le tamoxifen administré à des mammifères montre aussi certains effets œstrogéniques (18).

Alors que les doses d'œstrogènes et la cinétique d'induction ne sont pas les mêmes pour les deux protéines, la dose de tamoxifen à la demi-inhibition est la même. De plus, étudiant la décroissance du taux de synthèse de l'ovalbumine et de la conalbumine entre la $16^{\mathrm{e}}$ et la $24^{\mathrm{e}}$ heure après l'administration d'œstradiol, ainsi que celle du niveau du récepteur nucléaire de l'œstradiol, nous avons observé que les trois paramètres diminuaient parallèlement. Dans l'ensemble, on voit donc que les situations « déinduction » spontanée ou par le tamoxifen sont très semblables pour les deux protéines et par rapport au récepteur nucléaire, contrairement à l'absence de corrélation linéaire observée au cours de l'induction.

7) En administrant le tamoxifen après ou avant le traitement par l'œstrogène, les résultats ont indiqué deux principes importants. D'une part l'effet du tamoxifen semble virtuellement immédiat (fig. 3), l'arrêt de la synthèse dé la conalbumine en étant le test le plus facile à interpréter. Tout se passe comme si le tamoxifen, pourvu qu'il atteigne dans la cellule cible la concentration nécessaire (compte tenu de son affinité plus faible que celle de l'œstradiol pour le récepteur) peut remplacer le ligand œstrogène en suivant les lois habituelles de la compétition des ligands. D'autre part, en administrant le tamoxifen à des poulets recevant de l'œstradiol, on se met dans une situation où la majorité des récepteurs est déjà dans le noyau, inversement, quand on « relance » l'effet œstrogène en administrant de l'œstradiol benzoate à un animal sous tamoxifen, le récepteur (sous forme de complexe tamoxifen-récepteur) est également nucléaire. Cependant, dans les deux cas on observe, toujours en fonction des concentrations respectives de l'agoniste et de l'antagoniste, un effet qui indique l'accessibilité des sites récepteurs au ligand considéré quelle que soit sa localisation subcellulaire, et en particulier même si le récepteur est nucléaire. En d'autres termes, le rôle du récepteur cytoplasmique en tant qu'agent de transfert d'un ligand agoniste ou antagoniste dans le noyau nous semble une notion discutable. Ce qui compte, c'est que le ligand ait selon le cas une structure permettant au récepteur d'être actif ou au contraire l'empêchant d'être actif au niveau des sites (foujours inconnus) où se déclenche l'action.

8) L'induction de la synthèse de l'ovalbumine et de la conalbumine par la progestérone n'est pas inhibée par le tamoxifen, ce qui exclut un rôle important des œstrogènes endogènes dans le mécanisme de la réponse à la progestérone. Nous avons observé au contraire un résultat paradoxal, puisque l'injection simultanée de iamoxifen et de progestérone a donné constamment, tant pour la synthèse d'ovalbumine que celle de conalbumine, une potentialisation de la réponse observée avec la progestérone seule. Ce 


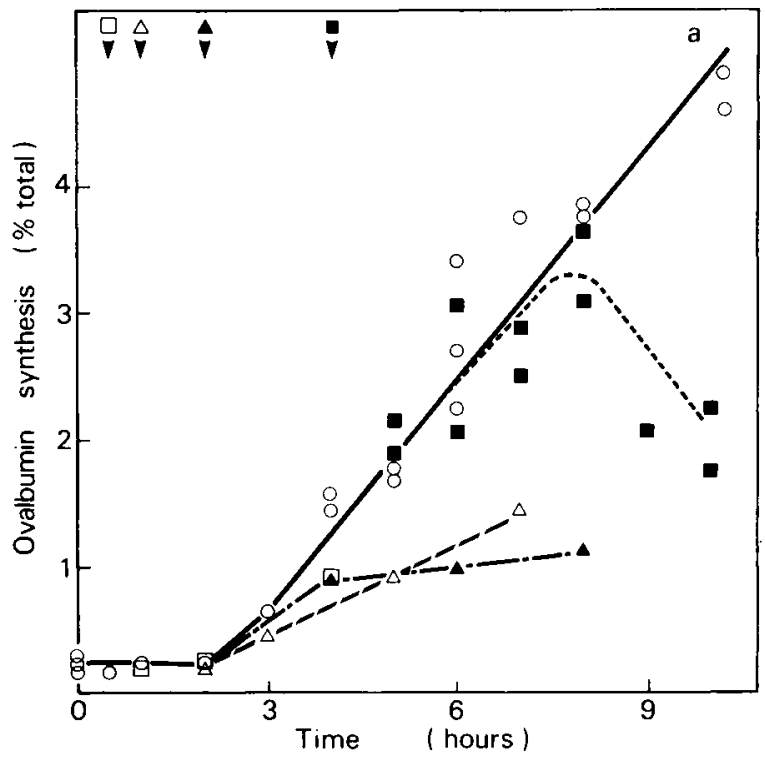

FIG. 3A.

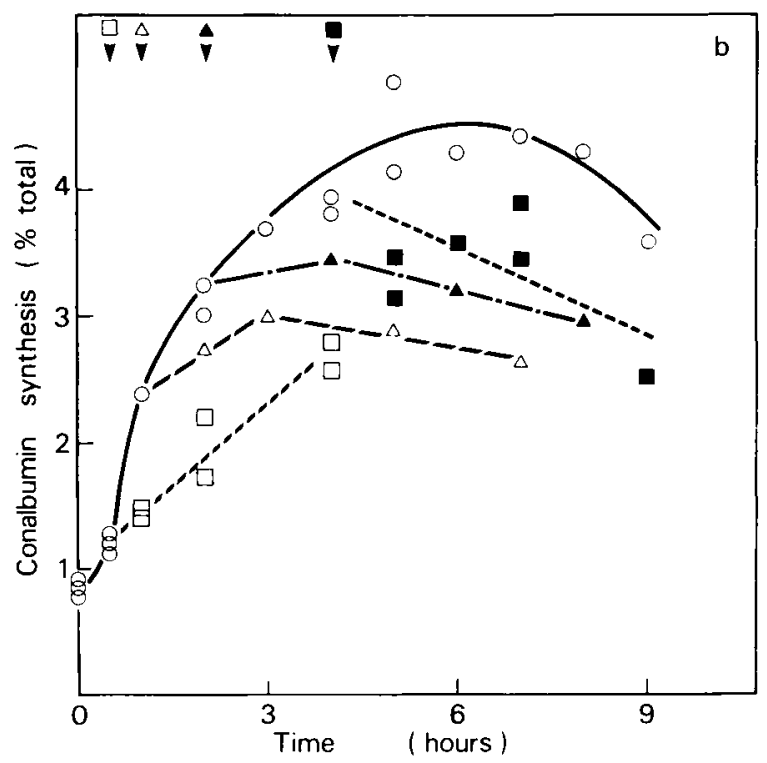

FIG. 3B.

FIG. 3. - Effet de l'administration retardée de tamoxifen sur l'induction précoce de la synthèse de l'ovalbumine ef de la conalbumine por l'œestradiol benzoale. Tous les poulets reçoivent $1 \mathrm{mg} / \mathrm{kg}$ d'œestradiol benzoate. Le tamoxifen $(10 \mathrm{mg} / \mathrm{kg})$ est injecté aux temps indiqués par les flèches, et le taux relatif de la synthèse de l'ovalbumine (A) et de la conalbumine (B) mesuré. Traitement par cestradiol benzoate seul (o) ou par tamoxifen injecté à $1 / 2 \mathrm{~h}(\mathrm{a}), 1 \mathrm{~h}(\Delta), 2 \mathrm{~h} \mathrm{( \Delta )}$, ou $1 \mathrm{~h}(\mathbf{(})$ après l'œstradiol benzoate. 
dernier effet tout à fait inattendu a été vérifié histologiquement, confirmé au cours de nombreuses expériences et chez l'animal très jeune non traité préalablement aux œstrogènes. Son mécanisme est en cours d'études.

En conclusion, ces recherches indiquent que l'oviducte de poulet permet des études de pharmacologie hormonale extrêmement détaillées, grâce à la précision des instruments immunologiques permettant des dosages précis du taux de synthèse des protéines. Les études en cours sur la structure des gènes des protéines du blanc de l'œuf dans les groupes de Chambon et O'Malley permettent également toute une série de nouvelles expériences portant sur le métabolisme des acides ribonucléiques, et des mécanismes de la transcription. Avec quelques nuances, le système n'apparaît pas différent de ce que l'on peut étudier chez les mammifères, et les résultats obtenus avec les hormones stéroïdes naturelles et de synthèse ainsi qu'avec les anti-hormones peuvent servir directement de fil conducteur pour étudier et expliquer la physiopathologie hormonale chez les mammifères et chez l'homme, tout en procurant aux investigateurs intéressés aux plans cellulaire et moléculaire des possibilités particulièrement favo. rables d'études d'encrinologie moléculaire.

Présenté ou Colloque D.G.R.S.T. de Port Bail, 27 février-1er mars 1979.

Accepté en octobre 1979.

\section{Références}

1. KOHLER P. O., GRIMLEY P. M., O'MALLEY B. W., 1969. Estrogen-induced cytodifferentiation of the ovalbumin-secreting glands of the chick oviduct. J. Cell Biol., 40, 8-27.

2. OKA T., SCHIMKE R. T., 1969. Interaction of estrogen and progesterone in chick oviduct development. I. Antagonist effect of progesterone on estrogen induced proliferation and differentiation of tubular gland cells. J. Cell Biol., 41, 816-831.

3. OKA T., SCHIMKE R. T., 1969. Interaction of estrogen and progesterone on chick oviduct development. II. Effects of estrogen and progesterone on tubular gland cell function. J. Cell Biol., 43, 123-137.

4. PALMITER R. D., MOORE P. B., MULVIHILL E. K., EMTAGE S., 1976. A significant lag in the induction of ovalbumin messenger RNA by steroid hormones : a receptor translocation hypothesis. Cell, 8, 557-572.

5. SCHÜTZ G., NGUYERO-MUU M. C., GIESECKE K., HYNES N. E., GRONER B., WURTZ T., SIPPEL A. E., 1977. Hormonal control of egg white protein messenger RNA synthesis in the chicken oviduct. Symp. on quantitative Biology, Vol. 42, part 2, Cold Spring Harbor Labor., 617-624.

6. PALMITER R. D., GUTMAN G. A., 1972. Fluorescent antibody localization of ovalbumin conalbumin, ovomucoid and lysozyme in chick oviduct magnum. J. biol. Chem., 247, 6459-6461.

7. MESTER J., BAULIEU E. E., 1977. Progesterone receptors in the chick oviduct. Determination of the total concentration of binding sites in the cytosol and nuclear fraction and effect of progesterone on their distribution. Eur. J. Biochem., 72, 405-414.

8. SUTHERLAND R. L., BAULIEU E. E., 1976. Quantitative estimates of cytoplasmic and nuclear estrogen receptor in chick oviduct. Eur. J. Biochem., 70, 531-541.

9. COX R. F., CATLIN G. H., CAREY N. H., 1971. Studies on the mode of action of estradiol on chicken oviduct. Characterisation of estradiol receptor proteins. Eur. J. Biochem., 22, 46-56.

10. SCHRADER W. T., O'MALLEY B. V., 1972. Progesterone binding components of chick oviduct IV. Characterisation of purified subunits. J. biol. Chem., 247, 51-59.

11. Mc KNIGHT G. S., PENNEQUIN P., SCHIMKE R. T., 1975. Induction of ovalbumin mRNA sequences by estrogen and progesterone in chick oviduct as measured by hybridization to complementary DNA. J. biol. Chem., 250, 8105-8110. 
12. SUTHERLAND R., MESTER J., BAULIEU E. E., 1977. Hormonal regulation of sex steroid hormone receptor concentration and subcellular distribution in chick oviduct. DUMONT J., and NUNEZ J., In Hormones and cell regulation. Vol. 1, 31-48, North Holland Publ. Co., Amsterdam.

13. SUTHERLAND R., MESTER J., BAULIEU E. E., 1977. Tamoxifen is a potent " pure » anti-estrogen in chick oviduct. Nature, 267, 434-435.

14. SUTHERLAND R., GEYNET C., BINART N., CATELLI MG., SCHMELK P. H., LEBEAU M. C., BAULIEU E. E., 1979. Steroid receptors and effects of estradiol and progesterone on chick oviduct proteins. Eur. J. Biochem. (soumis).

15. CATELLI MG., BINART N., ELKIK F., BAULIEU E. E. Effect of tamoxifen on esiradiol and progesterone induced synthesis of ovalbumin and conalbumin in chick oviduct. Eur. J. Biochem. (soumis).

16. PALMITER R. D., OKA T., SCHIMKE R. T., 1971. Modulation of ovalbumin synthesis by estradiol $17 \beta$ and actinomycine $D$ as studied in explants of chick oviduct in culture. J. biol. Chem., 246, 724-737.

17. PALMITER R. D., MULVIHILL E. R., Mc KNIGHT G. S., SENEAR A. W., 1977. Regulation of gene expression in the chick oviduct by steroid hormones. Symp. quantitative Biology, Vol. 42, part 2. Cold spring Harbor Labor., 639-647.

18. JORDAN V. C., DIX C. J., NAYLOR K. E., PRESTWICH G., ROWSBY L., 1978. Nonsteroidal antiestrogens : their biological effects and potential mechanisms of action. J. Toxicol. environ. Health, 4, 363-390. 